\title{
The expression levels of CD44v6 are correlated with the invasiveness of hepatocellular carcinoma in vitro, but do not appear to be clinically significant
}

\author{
KOSUKE MIMA, HIROHISA OKABE, TAKATSUGU ISHIMOTO, HIROMITSU HAYASHI, SHIGEKI NAKAGAWA, \\ HIDEYUKI KUROKI, KEISUKE MIYAKE, HIROSHI TAKAMORI, TORU BEPPU and HIDEO BABA \\ Department of Gastroenterological Surgery, Graduate School of Medical Sciences, \\ Kumamoto University, Kumamoto 860-8556, Japan
}

Received December 27, 2011; Accepted January 27, 2012

DOI: $10.3892 / \mathrm{ol} .2012 .611$

\begin{abstract}
The finding that the expression of a variant isoform of CD44 induced a metastatic phenotype in locally growing tumor cells has attracted considerable attention. A number of studies have analyzed the expression of CD44v6 in human tumors of different origins. However, the findings of these studies have been controversial. Therefore, in the present study, we assessed the association between CD44v6 expression and the invasive capacity of hepatocellular carcinoma (HCC) cell lines and also investigated the clinical significance of CD44v6 in $150 \mathrm{HCC}$ patients by immunohistochemical analysis. The HCC cell lines with a high CD44v6 expression, including HLF, HLE and SK HEP-1, showed high invasive potential, whereas those with a low CD44v6 expression, including PLC/PRF/5 and $\mathrm{HuH} 1$, showed low invasiveness. Despite these observations, we did not find any significant correlation between CD44v6 expression and clinicopathological factors in patients. By contrast, there was a weak correlation between a low CD44v6 expression and vascular invasion in $\mathrm{HCC}$ patients $(\mathrm{P}=0.080)$. Kaplan-Meier curves revealed that a high CD44v6 expression was not significantly associated with disease-free survival $(\mathrm{P}=0.736)$ or overall survival ( $\mathrm{P}=0.736)$. Our study suggests that the expression levels of CD44v6 are correlated with the invasiveness of HCC in vitro, but do not appear to be clinically significant. Future experiments should investigate the role of the various CD44 isoforms, including the CD44 standard isoform, in HCC cell lines and in patients with HCC.
\end{abstract}

Correspondence to: Dr Hideo Baba, Department of Gastroenterological Surgery, Graduate School of Medical Sciences, Kumamoto University, 1-1-1 Honjo, Kumamoto 860-8556, Japan E-mail: hdobaba@kumamoto-u.ac.jp

Key words: CD44, CD44v6, hepatocellular carcinoma

\section{Introduction}

Hepatocellular carcinoma (HCC) is the fifth most prevalent cancer and the third most common cause of cancer mortality worldwide (1,2). Surgical resection and liver transplantation are available for the treatment of early-stage HCC, but the prognosis of $\mathrm{HCC}$ remains poor due to the high level of tumor invasion, frequent intrahepatic spread, presence of extrahepatic metastasis and resistance to chemotherapy $(3,4)$. Although the introduction of sorafenib provided successful treatment for advanced HCC $(5,6)$, no established standard chemotherapeutic agents or molecular targeted agents are currently available for use as either neoadjuvant or adjuvant therapy. Additionally, sorafenib does not sufficiently eradicate cancer cells. Therefore, novel and more effective agents are required to improve the outcomes in patients with $\mathrm{HCC}$.

CD44, a major adhesion molecule for the extracellular matrix, is involved in a wide variety of physiological processes, including leukocyte homing and activation, wound healing and cell migration $(7,8)$. Through alternative mRNA splicing, cells produce numerous CD44 protein isoforms. The standard isoform (CD44s) is expressed predominantly in hematopoietic cells and normal epithelial cell subsets, whereas variant isoforms (CD44v) are expressed by certain epithelial cells, during embryonic development, lymphocyte maturation and activation and in several types of carcinoma. The finding that the expression of a CD44v induced a metastatic phenotype in locally growing tumor cells attracted considerable interest (9). Expression of the CD44v6 isoform was detected in patients with high-grade non-Hodgkin's lymphoma and found to correlate with a poor prognosis (10). In colorectal cancer patients, the expression of CD44s and the CD44v correlated with a poor prognosis and may be considered as a strong prognostic factor in patients (11). In patients with cervical cancer, a high level of expression of CD44v6 is associated with a poor prognosis (12). Similarly, in breast cancer patients, the expression of variants containing the exons v3, v6 and v7/8 correlates with more aggressive stages of the disease (13). However, there are certain tumor types, including neuroblastomas and prostate cancer, in which the absence of CD44 expression correlates with transformation and a poor prognosis $(14,15)$. 
As such, while numerous studies have analyzed the expression of CD44 isoforms in human tumors of different origins, their results were often controversial.

Therefore, we assessed the association between CD44v6 and the invasive capacity of human HCC cell lines. The clinical significance of CD44v6 was also investigated by immunohistochemical analysis in patients with HCC.

\section{Materials and methods}

Cell lines and culture conditions. The PLC/PRF/5, HuH1, HLF and HLE human HCC cell lines were purchased from the Japanese Collection of Research Bioresources (Osaka, Japan). SK HEP-1 was purchased from the American Type Culture Collection (Manassas, VA, USA). The cells were routinely maintained in Dulbecco's modified Eagle's medium (Invitrogen, Carlsbad, CA, USA) supplemented with $10 \%$ fetal bovine serum (FBS; Invitrogen). The cells were incubated at $37^{\circ} \mathrm{C}$ in a $5 \% \mathrm{CO}_{2}$ air-humidified atmosphere.

Protein extraction and western blot analysis. The protein extraction from cultivated cells and the western blot analyses were performed as previously described $(16,17)$. In brief, the cells were lysed in a cell lysis buffer containing $25 \mathrm{mM}$ Tris (pH 7.4), $100 \mathrm{mM} \mathrm{NaCl}$ and 1\% Tween-20. Equal amounts of the proteins were loaded onto $10 \%$ gels and separated by SDS-PAGE. The resolved proteins were electrophoretically transferred to polyvinylidene fluoride (PVDF) membranes (Bio-Rad, Inc., Hercules, CA, USA). The membranes were blocked with 5\% low-fat dry milk in TBS-T [25 mM Tris ( $\mathrm{pH} 7.4$ ), $125 \mathrm{mM} \mathrm{NaCl}, 0.4 \%$ Tween-20] for $1 \mathrm{~h}$ at room temperature, followed by overnight incubation with the primary antibody at $4^{\circ} \mathrm{C}$. The blots were extensively washed with TBS-T and incubated at a 1:2000 dilution of HRP-conjugated secondary antibody (Santa Cruz Biotechnology and Cell Signaling, Santa Cruz, CA, USA) diluted in TBS-T for $1 \mathrm{~h}$ at room temperature. The membranes were washed and visualized using a chemiluminescent detection reagent kit (ECL; GE Healthcare Corp., Piscataway, NJ, USA). A primary antibody against CD44v6 (1:1000 dilution; Bender Medsystems, San Diego, CA, USA) was used in this study.

RNA extraction and quantitative RT-PCR. Total RNA extraction, complementary DNA (cDNA) synthesis and quantitative real-time PCR were performed as described previously $(16,17)$. The total RNA was extracted from the cells using the RNeasy Mini kit (Qiagen, Hilden, Germany) and cDNA was synthesized with the SuperScript III transcriptor first strand cDNA synthesis system for RT-PCR (Invitrogen), according to the manufacturer's instructions. Quantitative reverse transcription PCR (qRT-PCR) was performed using a LightCycler 480 II instrument (Roche, Mannheim, Germany). To determine the difference in the gene expression levels between the specimens, $2^{-\Delta \Delta C t}$ was used to measure the fold changes among the samples (18). To perform qRT-PCR, primers were designed using the Universal Probe Library (Roche) according to the manufacturer's instructions. The primer sequences used in the real-time PCR were: CD44, 5'-GCAGTCAACAGTCGAAGAAGG-3', 5'-TGTCCTCCACAGCTCCATT-3' and universal probe no. 29; and GAPDH, 5'-AGCCACATCGCTCAGACAC-3', 5'-GCCCAATACGACCAAATCC-3' and universal probe no. 60 .

Cell invasion assay. The in vitro cell invasion assay was performed as previously described (17). In brief, the invasion of tumor cells that migrated through transwell inserts with a uniform layer of BD Matrigel basement membrane matrix (BD Biosciences) was assessed according to the manufacturer's instructions. The cells were seeded at $5 \times 10^{4}$ in $500 \mu \mathrm{l}$ of serum-free medium in the upper chamber of the insert and medium containing 10\% FBS was added in the lower chamber. After $22 \mathrm{~h}$, the non-invading cells were removed with a cotton swab, the invading cells were stained with $1 \%$ toluidine blue and the cells were counted under a microscope.

Patients and treatment. From the 235 consecutive patients who had undergone curative hepatic resection between 2004 and 2007 in the Department of Gastroenterological Surgery, Graduate School of Medical Sciences (Kumamoto University, Kumamoto, Japan), 150 primary HCC samples were analyzed in this study. None of the patients had received any preoperative anticancer treatment. The pathological diagnoses and the clinicopathological factors of patients were established based on the general rules of primary liver cancer of the Liver Cancer Study Group of Japan $(19,20)$ and the American Joint Committee on Cancer (AJCC)/International Union Against Cancer (UICC) staging system (21). The median duration of follow-up after surgery was 44 months. This study was approved by the Human Ethics Review Committee of the Graduate School of Medicine, Kumamoto University (Kumamoto, Japan).

Immunohistochemistry (IHC) and scoring. The sample processing and IHC procedures were performed as previously described (17). Endogenous peroxidase activity was blocked using $3 \%$ hydrogen peroxide. The sections were incubated in diluted antibodies. A subsequent reaction was performed with a biotin-free HRP enzyme-labeled polymer from the Envision Plus detection system (Dako Co., Carpinteria, CA, USA). A positive reaction was visualized with a 3,3'-diaminobenzidine solution, followed by counterstaining with Meier's hematoxylin. The primary antibody for CD44v6 (1:100 dilution; Bender Medsystems) was used for the study. IHC staining was independently scored by two pathologists. For membranous CD44v6, the results were classified ranging from 0 to $3+$ : 0 , no staining; $1+, 1-25 \% ; 2+, 26-50 \%$; and $3+,>50 \%$ staining of the specimen. The 1,2 and 3+ specimens were considered to be positive IHC results.

Statistical analysis. The data are presented as the mean \pm SD. Independent Student's t-tests were used to compare the continuous variables between the two groups. Categorical variables were compared using the $\chi^{2}$ test. The overall and disease-free survival were calculated using the Kaplan-Meier method and compared using the log-rank test. The statistical analyses were performed as indicated with a statistical analysis software program (Excel Statistics, Social Survey Research Information Co., Tokyo, Japan). $\mathrm{P}<0.05$ was considered to indicate a statistically significant result. 
A

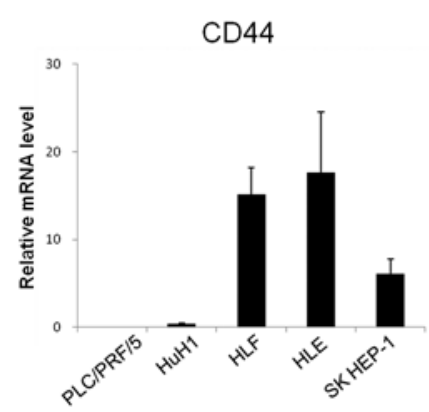

B

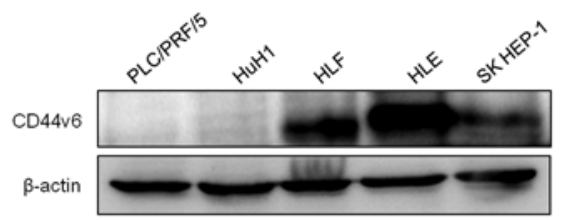

C

Figure 1. Expression profile of CD44v6 and the invasive properties in five HCC cell lines is shown. (A) Relative expression level of $C D 44 \mathrm{mRNA}$ in five HCC cell lines. The data are presented as the mean $\pm \mathrm{SD}(\mathrm{n}=3)$. (B) Expression of the CD44v6 proteins in the five HCC cell lines as determined by western blot analysis. (C) The invasive properties of the five HCC cell lines. The data are presented as the mean \pm SD $(n=3)$. CD44v6, splice variant 6 of CD44; HCC, hepatocellular carcinoma.
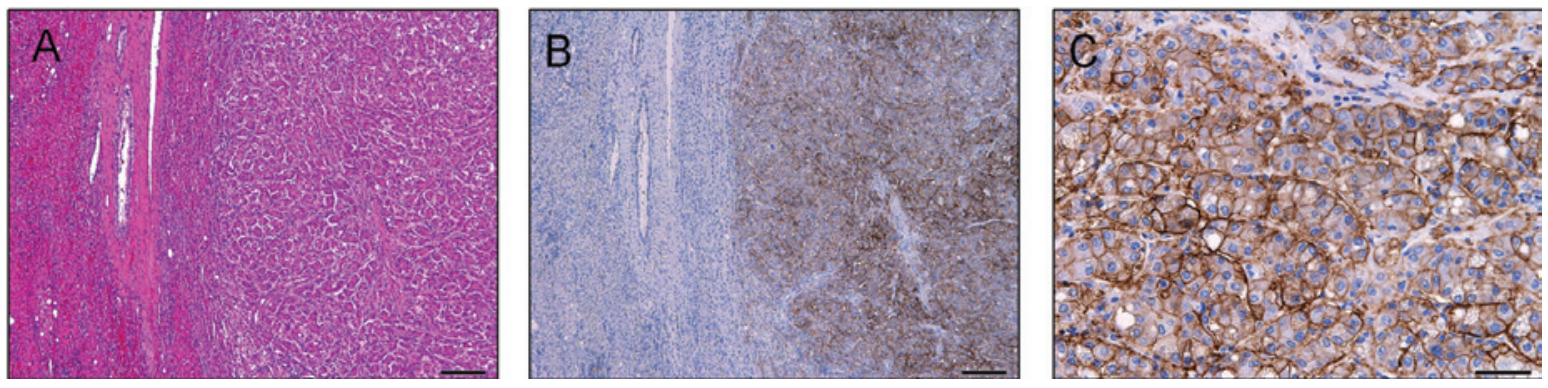

Figure 2. Expression of CD44v6 in hepatocellular carcinoma tissue specimens (membranous). (A) Hematoxylin and eosin staining of hepatocellular carcinoma. Scale bar, $200 \mu \mathrm{m}$. (B) Immunohistochemical staining of hepatocellular carcinoma. Scale bar, $200 \mu \mathrm{m}$. (C) Immunohistochemical staining of hepatocellular carcinoma in a high-power field. Scale bar, $50 \mu \mathrm{m}$. CD44v6, splice variant 6 of CD44.

\section{Results}

The expression of CD44v6 is associated with the invasive phenotype of HCC cells. We examined the expression of CD44v6 and its ability for invasiveness in five HCC cell lines (PLC/PRF/5, HuH1, HLF, HLE and SK HEP-1). The HLF, HLE and SK HEP-1 cells showed a high expression of CD44, while the PLC/PRF/5 and HuH1 cells showed a low expression of CD44 at the mRNA level (Fig. 1A). The mRNA levels in the cells corresponded to the protein levels, with cells that had high levels of CD44 mRNA also showing a high expression of the CD44v6 protein (Fig. 1B). The HLF, HLE and SK HEP-1 cells showed high invasiveness, whereas the PLC/PRF/5 and HuH1 cells showed low invasiveness (Fig. 1C). These results suggest that CD44v6 expression is correlated with the invasive phenotype in HCC cells.

The expression of CD44v6 in hepatocellular carcinoma. To investigate the clinical significance of the expression of CD44v6, we analyzed the protein levels of CD44v6 in the tumor and the adjacent liver tissue by IHC in samples from $150 \mathrm{HCC}$ patients. CD44v6 was mainly expressed in the tumor cell membrane (Fig. 2). A total of 46 cases (30.7\%) were diagnosed as positive for CD44v6 expression (Table I). CD44v6 was not expressed in the adjacent liver tissue in any of the patients (Table I).

The clinical significance of CD44v6 in hepatocellular carcinoma. No significant correlation was found between the level
Table I. Expression of CD44v6 in the tumors and adjacent liver sections of $150 \mathrm{HCC}$ patients.

\begin{tabular}{lcrcr}
\hline & \multicolumn{4}{c}{ CD44v6 score } \\
\cline { 2 - 5 } Tissue & 0 & $1+$ & $2+$ & $3+$ \\
\hline Tumor & 104 & 32 & 9 & 5 \\
Adjacent liver & 150 & 0 & 0 & 0
\end{tabular}

0 , no staining; $1+, 1-25 \% ; 2+, 26-50 \%$; and $3+,>50 \%$ staining of the specimen. CD44v6, splice variant 6 of CD44; HCC, hepatocellular carcinoma.

of CD44v6 expression and the clinicopathological factors (Table II). However, results showed a weak correlation between a low level of CD44v6 expression and vascular invasion in HCC patients $(\mathrm{P}=0.080)$. Kaplan-Meier curves revealed that a high CD44v6 expression was not significantly associated with disease-free survival ( $\mathrm{P}=0.736$, Fig. $3 \mathrm{~A})$ or overall survival $(\mathrm{P}=0.736$, Fig. 3B).

\section{Discussion}

In the present study, the expression CD44v6 was found to be correlated with the invasive phenotype of HCC cell lines. 
Table II. Correlation between the expression of CD44v6 and the clinicopathological factors of 150 HCC patients.

\begin{tabular}{|c|c|c|c|}
\hline Clinicopathological factors & $\begin{array}{c}\text { CD44v6 positive } \\
(\mathrm{n}=46)\end{array}$ & $\begin{array}{l}\text { CD44v6 negative } \\
\quad(\mathrm{n}=104)\end{array}$ & P-value \\
\hline Age $\leq 60 />60$ (years) & $10 / 36$ & $34 / 70$ & 0.174 \\
\hline Male/female & $38 / 8$ & $85 / 19$ & 0.897 \\
\hline HBs-Ag negative/positive & $32 / 14$ & $75 / 29$ & 0.750 \\
\hline HCV-Ab negative/positive & $23 / 23$ & $58 / 46$ & 0.513 \\
\hline Child-Pugh classification A/B & $41 / 5$ & $94 / 10$ & 0.813 \\
\hline $\mathrm{AFP} \leq 20 />20(\mathrm{ng} / \mathrm{ml})$ & $24 / 22$ & $52 / 52$ & 0.806 \\
\hline PIVKA-II $\leq 107 />107$ (mAU/ml) & $24 / 22$ & $51 / 53$ & 0.723 \\
\hline Tumor size $\leq 3 />3(\mathrm{~cm})$ & $16 / 30$ & $34 / 70$ & 0.802 \\
\hline Tumor number $1 / \geq 2$ & $33 / 13$ & $73 / 31$ & 0.848 \\
\hline Tumor encapsulation absent/present & $5 / 41$ & $12 / 92$ & 0.905 \\
\hline Tumor differentiation moderate, well/poor & $37 / 9$ & $84 / 20$ & 0.962 \\
\hline LCSGJ TNM stage $1,2 / 3,4$ & $27 / 19$ & $62 / 42$ & 0.916 \\
\hline AJCC/UICC TNM stage $1,2 / 3,4$ & $40 / 6$ & $82 / 22$ & 0.240 \\
\hline Vascular invasion absent/present & $45 / 1$ & $93 / 11$ & 0.080 \\
\hline
\end{tabular}

Vascular invasion, portal vein (3rd branch, 2nd branch, 1st branch or trunk) invasion and hepatic vein (trunk of hepatic vein or IVC) invasion were defined by a macroscopic examination of the resected specimens. HBs-Ag, hepatitis B surface antigen; HCV-Ab, hepatitis C antibody; AFP, $\alpha$-fetoprotein; PIVKA-II, protein-induced vitamin K absence-II; LCSGJ, Liver Cancer Study Group of Japan; AJCC/UICC, American Joint Committee on Cancer/International Union Against Cancer; CD44v, splice variant 6 of CD44; HCC, hepatocellular carcinoma.

A

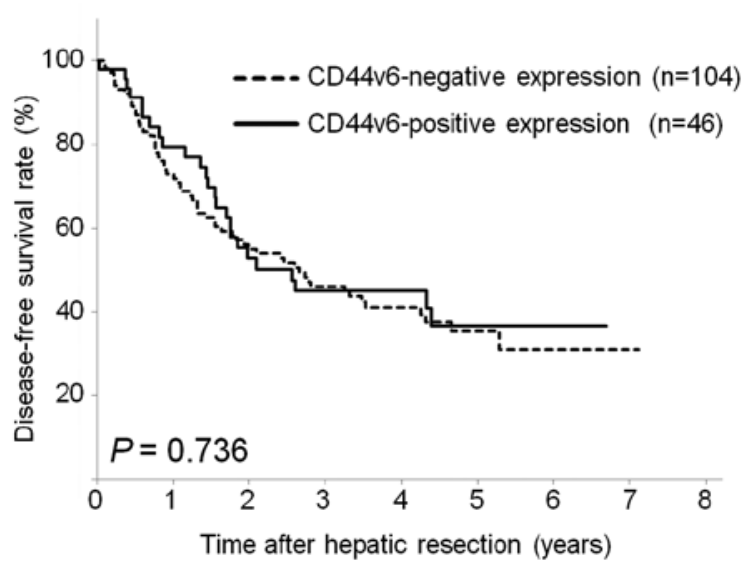

B

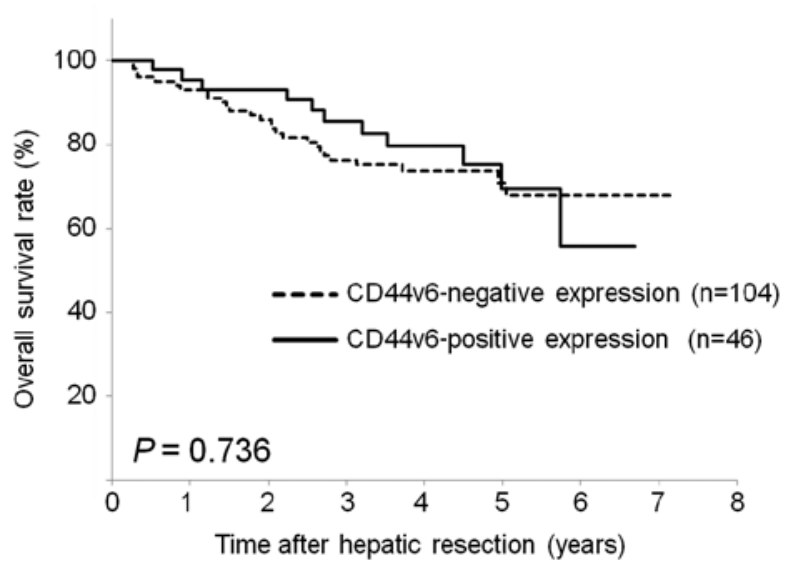

Figure 3. Kaplan-Meier survival analysis of (A) disease-free and (B) overall survival in HCC patients comparing those positive for CD44v6 expression with those negative for CD44v6 expression, as compared using the log-rank test. HCC, hepatocellular carcinoma; CD44v6, splice variant 6 of CD44.

However, the level of CD44v6 expression was not found to be correlated with the survival or clinicopathological factors of patients with HCC. Instead, a low expression level of CD44v6 tended to be associated with vascular invasion.

In a previous study, Endo and Terada reported that a high CD44v6 expression in patients with $\mathrm{HCC}$ was significantly correlated with the presence of vascular invasion and a poor prognosis (22), which was not in agreement with our results. Both the present study and that by Endo and Terada analyzed the expression of CD44v6 by IHC in samples from patients with HCC following hepatectomy. The proportion of CD44v6positive cases in our study was similar to that in the study of Endo and Terada (30.6 vs. 34\%). However, these authors did not report the characteristics of the patients. Therefore, the discrepancy in the results may be due to differences in the characteristics of the patients included in the studies.

CD44v6 acts as an essential co-receptor for the activation of Met. In several cell lines, CD44v6 catalyzes the formation of a complex with Met and its ligand (23). In addition, CD44v6 binding to the extracellular matrix also activates the PI3K-Akt pathway and regulates Met transcription. These observations suggest that the role of CD44v6 in the invasive and malignant phenotype are linked to its collaboration with receptor tyrosine kinases and c-Met. However, the CD44s isoform is essential for the response to TGF- $\beta$ in the epithelialmesenchymal transition (EMT) and an increase in CD44s 
expression was found to be accompanied by a loss of variant isoforms in breast cancer (24). The EMT is a developmental process in which epithelial cells lose their polarity and acquire the migratory properties of mesenchymal cells. The EMT has been shown to be a pivotal mechanism in cancer invasion and metastasis $(25,26)$.

In conclusion, our study suggests that the expression level of CD44v6 is correlated with the invasiveness of hepatocellular carcinoma in vitro, but does not appear to be clinically significant. Instead, a low expression of CD44v6 showed a tendency to be associated with the vascular invasion of HCC. Future experiments should investigate the role of the various CD44 isoforms, including the CD44s isoform, in HCC cell lines and in patients with HCC.

\section{References}

1. Jou J and Diehl AM: Epithelial-mesenchymal transitions and hepatocarcinogenesis. J Clin Invest 120: 1031-1034, 2010.

2. Jemal A, Siegel R, Ward E, Hao Y, Xu J and Thun MJ: Cancer statistics. CA Cancer J Clin 59: 225-249, 2009.

3. Poon RT, Ng IO, Fan ST, et al: Clinicopathologic features of long-term survivors and disease-free survivors after resection of hepatocellular carcinoma: a study of a prospective cohort. J Clin Oncol 19: 3037-3044, 2001

4. Eguchi S, Kanematsu T, Arii S, et al: Recurrence-free survival more than 10 years after liver resection for hepatocellular carcinoma. Br J Surg 98: 552-557, 2011.

5. Llovet JM, Ricci S, Mazzaferro V, et al: Sorafenib in advanced hepatocellular carcinoma. N Engl J Med 359: 378-390, 2008

6. Villanueva A and Llovet JM: Targeted therapies for hepatocellular carcinoma. Gastroenterology 140: 1410-1426, 2011.

7. Ponta H, Sherman L and Herrlich PA: CD44: from adhesion molecules to signalling regulators. Nat Rev Mol Cell Biol 4: 33-45, 2003.

8. Zöller M: CD44: can a cancer-initiating cell profit from an abundantly expressed molecule? Nat Rev Cancer 11: 254-267, 2011.

9. Günthert U, Hofmann M, Rudy W, et al: A new variant of glycoprotein CD44 confers metastatic potential to rat carcinoma cells. Cell 65: 13-24, 1991.

10. Stauder R, Eisterer W, Thaler J and Günthert U: CD44 variant isoforms in non-Hodgkin's lymphoma: a new independent prognostic factor. Blood 85: 2885-2899, 1995.
11. Wielenga VJ, van der Neut R, Offerhaus GJ and Pals ST: CD44 glycoproteins in colorectal cancer: expression, function, and prognostic value. Adv Cancer Res 77: 169-187, 2000.

12. Kainz C, Kohlberger P, Tempfer C, et al: Prognostic value of CD44 splice variants in human stage III cervical cancer. Eur J Cancer 31A: 1706-1709, 1995.

13. Götte M and Yip GW: Heparanase, hyaluronan, and CD44 in cancers: a breast carcinoma perspective. Cancer Res 66: 10233-10237, 2006.

14. Shtivelman E and Bishop JM: Expression of CD44 is repressed in neuroblastoma cells. Mol Cell Biol 11: 5446-5453, 1991.

15. Gao AC, Lou W, Dong JT and Isaacs JT: CD44 is a metastasis suppressor gene for prostatic cancer located on human chromosome 11p13. Cancer Res 57: 846-849, 1997.

16. Hiyoshi Y, Kamohara H, Karashima R, et al: MicroRNA-21 regulates the proliferation and invasion in esophageal squamous cell carcinoma. Clin Cancer Res 15: 1915-1922, 2009.

17. Okabe H, Beppu T, Hayashi $\mathrm{H}$, et al: Hepatic stellate cells accelerate the malignant behavior of cholangiocarcinoma cells. Ann Surg Oncol 18: 1175-1184, 2011.

18. Livak KJ and Schmittgen TD: Analysis of relative gene expression data using real-time quantitative PCR and the 2(-Delta Delta C(T)) Method. Methods 25: 402-408, 2001.

19. Liver Cancer Study Group of Japan: The General Rules for the Clinical and Pathological Study of Primary Liver Cancer. Kanehara, Tokyo, 2009.

20. Minagawa M, Ikai I, Matsuyama Y, Yamaoka Y and Makuuchi M: Staging of hepatocellular carcinoma: assessment of the Japanese TNM and AJCC/UICC TNM systems in a cohort of 13,772 patients in Japan. Ann Surg 245: 909-922, 2007.

21. Vauthey JN, Lauwers GY, Esnaola NF, et al: Simplified staging for hepatocellular carcinoma. J Clin Oncol 20: 1527-1536, 2002.

22. Endo K and Terada T: Protein expression of CD44 (standard and variant isoforms) in hepatocellular carcinoma: relationships with tumor grade, clinicopathologic parameters, p53 expression, and patient survival. J Hepatol 32: 78-84, 2000.

23. Orian-Rousseau V, Chen L, Sleeman JP, Herrlich P and Ponta H: CD44 is required for two consecutive steps in $\mathrm{HGF} / \mathrm{c}-\mathrm{Met}$ signaling. Genes Dev 16: 3074-3086, 2002.

24. Brown RL, Reinke LM, Damerow MS, et al: CD44 splice isoform switching in human and mouse epithelium is essential for epithelial-mesenchymal transition and breast cancer progression. J Clin Invest 121: 1064-1074, 2011.

25. Polyak K and Weinberg RA: Transitions between epithelial and mesenchymal states: acquisition of malignant and stem cell traits. Nat Rev Cancer 9: 265-273, 2009.

26. Thiery JP, Acloque H, Huang RY and Nieto MA: Epithelialmesenchymal transitions in development and disease. Cell 139: 871-890, 2009. 